\title{
悬 \\ Comparação entre métodos para o cálculo de avanço da tuberosidade tibial em cães: estudo em 80 joelhos
}

[Comparison of methods for calculating tibial tuberosity advancement in dogs: a study of 80 knees]

\section{"Artigo Científico/Scientific Article"}

\author{
Maria Isabel Rocha Sampaio ${ }^{1}$, Fabiano Sellos Costa $^{2}$, Sabrina Cândido Trajano ${ }^{2}$, Eduardo Alberto \\ Tudury $^{2 *}$
}

${ }^{1}$ Clínica 4 patas, Olinda-PE, Brasil.

${ }^{2}$ Departamento de Medicina Veterinária, Universidade Federal Rural de Pernambuco, Recife-PE, Brasil.

*Autor para correspondência/Corresponding author: E-mail: respeit@hotmail.com

\begin{abstract}
Resumo
Uma das técnicas cirúrgicas atuais mais populares para o tratamento da ruptura do ligamento cruzado cranial é o avanço da tuberosidade tibial (TTA). Esta pesquisa teve como objetivo comparar cinco métodos de cálculo desse avanço descritos por Dennler et al. (2006), Hoffmann et al. (2006), Vezzoni (2010), Ness (2011) e Koch (2016), verificando entre os dois joelhos do mesmo animal, se a quantidade de avanço necessária seria a mesma. Para isso os dois joelhos de 40 cães foram radiografados. No presente estudo observou-se que não há diferença significante entre os métodos de Hoffmann, de Ness e de Koch, porém há diferença entre tais métodos e os métodos de Dennler e de Vezzoni pré estabelecidas. Houve também índice de confiança moderado ao comparar o método do quadro pré definido com todos os outros métodos, assim como o da tangente comum com todos os outros métodos, exceto o do platô tibial, que demonstrou um índice de confiabilidade bom. Tal resultado positivo também foi observado ao comparar os demais métodos entre si. Quanto ao lado, não foi observada diferença significante entre membros direito e esquerdo, exceto no método descrito por Ness (2011) $(\mathrm{p}=0,038)$.
\end{abstract}

Palavras-chave: articulação femoro-tibio-patelar; ortopedia veterinária; tuberosidade da tíbia.

\begin{abstract}
One of the most popular current surgical techniques for the treatment of cranial cruciate ligament rupture is tibial tuberosity advancement (TTA). The main objectives were to compare the advancement calculation methods described by Dennler et al. (2006), Hoffmann et al. (2006), Vezzoni (2010), Ness (2011) and Koch (2016), also checking between the two stifles of the same animal. For this, 80 knees from 40 dogs were used. It was observed that there is no significant difference between the Hoffmann, Ness and Koch methods, but there is a difference between such methods and the Dennler and Vezzoni methods. There was also a moderate confidence index when comparing the pre-defined frame method with all other methods, as well as the common tangent with all other methods except the tibial plateau method, which demonstrated a good reliability index. This positive result was also observed when comparing the other methods among themselves. As to the side, no significant difference was observed between right and left limbs, except in the method described by Ness (2011) $(\mathrm{p}=0.038)$.
\end{abstract}

Keywords: femoro-tibio-patellar joint; veterinary orthopedics; tibial tuberosity.

\section{Introdução}

A TTA (Avanço da Tuberosidade Tibial) é uma técnica cirúrgica dinâmica que promove estabilidade para o suporte de peso, alterando a conformação da tíbia proximal, por meio de uma osteotomia da tuberosidade tibial no plano frontal e o avanço deste fragmento (Apelt, 2007; Miller, 2007), fazendo com que o ligamento patelar fique perpendicular à reta correspondente ao platô tibial. Esse posicionamento promove a diminuição 
da força de subluxação aplicada na posição de apoio do membro com extensão do joelho diminuindo o estresse no ligamento patelar (Kuhn et al., 2011; Kowaleski et al., 2017).

Para se atingir o avanço necessário précalculado por diversos métodos, se interpõe um espaçador (cage), devendo o elemento implantado possuir a medida adequada para que o ligamento patelar fique 90 graus em relação ao platô tibial (Zani et al., 2011; Meeson et al., 2018). Para se calcular a medida certa existem diversos métodos de cálculo (Dennler et al., 2006; Hoffmann et al 2006, Vezzoni, 2010; Ness, 2011; Koch, 2016).

Para alguns métodos de cálculo se faz necessário o ângulo de 135 graus entre tíbia e fêmur, como ocorre nos métodos do platô tibial e da tangente comum, os quais exigem visualização de fêmur distal e de toda a tíbia na imagem radiográfica para sua realização (Kowaleski et al., 2017).

No método de Ness (2011), se faz necessário somente a visualização de toda a tíbia e do tálus. Apesar de ter como vantagem não utilizar o fêmur, esta técnica apresenta passos a mais a serem realizados quando comparado às outras, podendo aumentar as chances de erro. $\mathrm{O}$ método de Koch (2016) utiliza apenas a tíbia proximal.

Objetivou-se verificar a concordância entre cinco métodos de cálculo de avanço da tuberosidade da tíbia, assim como, comparar entre os dois joelhos do mesmo animal se a quantidade de avanço necessária seria a mesma.

\section{Material e Métodos}

Para o experimento foram utilizados exames radiográficos de 80 joelhos de 40 animais da espécie canina, provenientes do atendimento de rotina de um centro de diagnóstico por imagem digital, sem distinção de sexo, raça ou idade, que não apresentassem ruptura do ligamento cruzado cranial ou qualquer anormalidade em tíbia que impossibilitasse os cálculos de avanço da tuberosidade tibial.

Animais que apresentassem rotação ou deformação da tíbia, ângulo do platô tibial maior que 30 graus, porção proximal da tíbia muito larga no sentido crânio caudal e cães muito pesados, que não se enquadrassem nos padrões para a realização da técnica cirúrgica TTA, também não entraram no estudo.

\section{Cálculos de Avanço}

A quantidade de avanço necessária foi determinada de modo que o ângulo do ligamento patelar alvo fosse igual a 90 graus em relação à linha do plato tibial medial. Métodos diferentes foram então utilizados para determinar este avanço. Para que todos eles fossem possíveis de serem efetuados, as radiografias foram realizadas na projeção médio lateral, com o joelho no ângulo de 135 graus e, com toda a tíbia e tálus inclusas na imagem radiográfica. Para todas as técnicas, ficou definido como ponto de inserção na tíbia do ligamento patelar a proeminência dorsal sobresalente da mesma, no local onde as fibras de Sharpey desencadeiam maior radiopacidade (Figura 1).

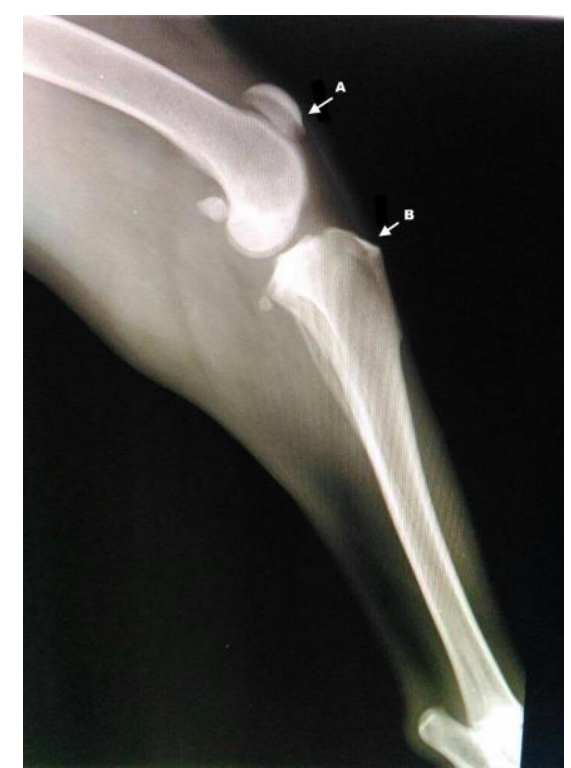

Figura 1. Ponto de inserção do ligamento patelar na patela (A) e na tuberosidade da tíbia (B).

\section{Método do Platô Tibial (Hoffmann et al. 2006))}

Com o programa Workstation Fujifilm, utilizando as ferramentas de régua e ângulo, foi desenhada nas imagens radiográficas, uma reta unindo a extremidade cranial e caudal do côndilo medial da tíbia extendendo cranialmente, e uma reta perpendicular a esta, partindo da inserção do ligamento patelar na patela, sendo a distância entre a segunda reta e a crista tibial ao nível da inserção do ligamento patelar a medida necessária do espaçador a implantar caso o animal apresente RLCCr (Figura 2).

\section{Método da Tangente Comum (Dennler et al., 2006)}

Utilizando as ferramentas de régua, ângulo e círculos do programa, foi desenhado na imagem 
radiográfica círculos que correspondem às respectivas superfícies articulares dos côndilos femorais e do platô tibial; passando o círculo femoral pela fossa extensora e os lados do círculo tibial passando pelas extremidades do platô tibial medial. Em seguida foi desenhada uma reta unindo o centro dos dois círculos e outra reta formando um ângulo de 90 graus com a primeira. Logo, uma terceira reta foi realizada vindo da inserção do ligamento patelar na patela formando um ângulo de 90 graus com a segunda reta. A distância entre esta terceira reta e a inserção do ligamento patelar na tíbia corresponde à quantidade do avanço necessária (Figura 3).

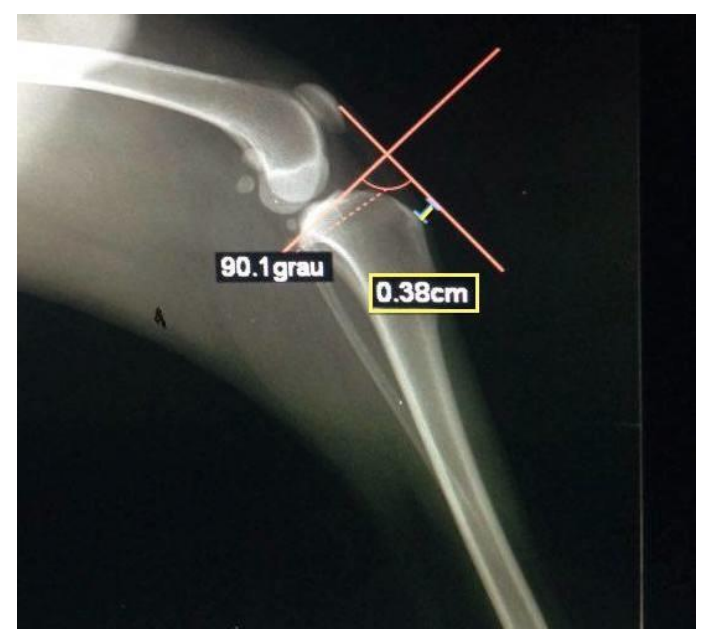

Figura 2. Método do platô tibial para cálculo de avanço para TTA.

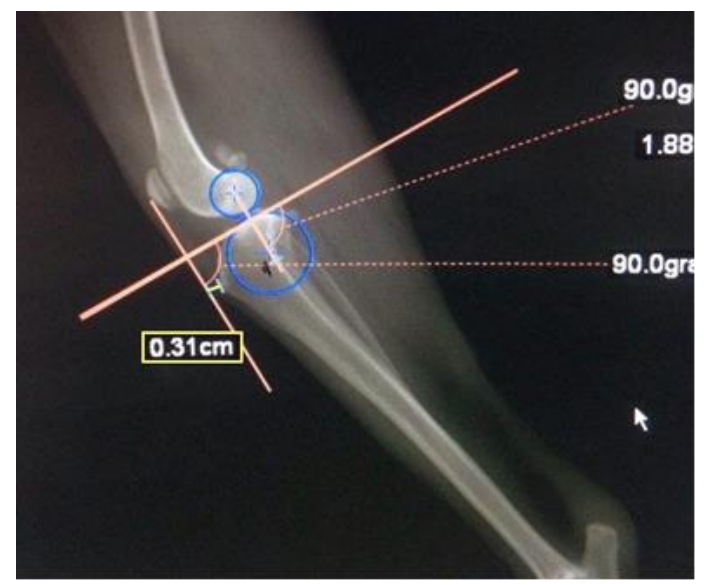

Figura 3. Método da Tangente comum para cálculo de avanço para TTA.

\section{Método de Ness (2011)}

Para o método de Ness, foi traçada nas radiografias uma reta $\mathrm{AB}$ através do platô tibial se extendendo cranial e caudalmente; localizado a eminência intercondilar e o ponto central do tálus foi traçada uma linha $\mathrm{CD}$; do ponto $\mathrm{C}$, foi traçada uma nova reta $\mathrm{CE}$ que formasse 135 graus com a reta $\mathrm{CD}$; em seguida localizada a inserção do ligamento patelar e traçada a partir deste ponto uma reta FG de modo que fosse paralela a CE. Foi então localizado o ponto $\mathrm{H}$, onde $\mathrm{FG}$ cruza $\mathrm{AB}$, desenhado uma reta $\mathrm{FI}$, para cruzar $\mathrm{AB}$ no ponto $\mathrm{J}$, onde FJA fosse um ângulo reto, em seguida desenhado uma reta FL que fosse perpendicular a $\mathrm{CD}$, depois foi desenhada uma reta que cruzasse $\mathrm{H}$ e que fosse paralela a IF e que cruzasse FL no ponto M. Foi então, medida a distância entre $\mathrm{F}$ e $\mathrm{M}$, e esta distância é a requerida para o avanço (Figura 4).

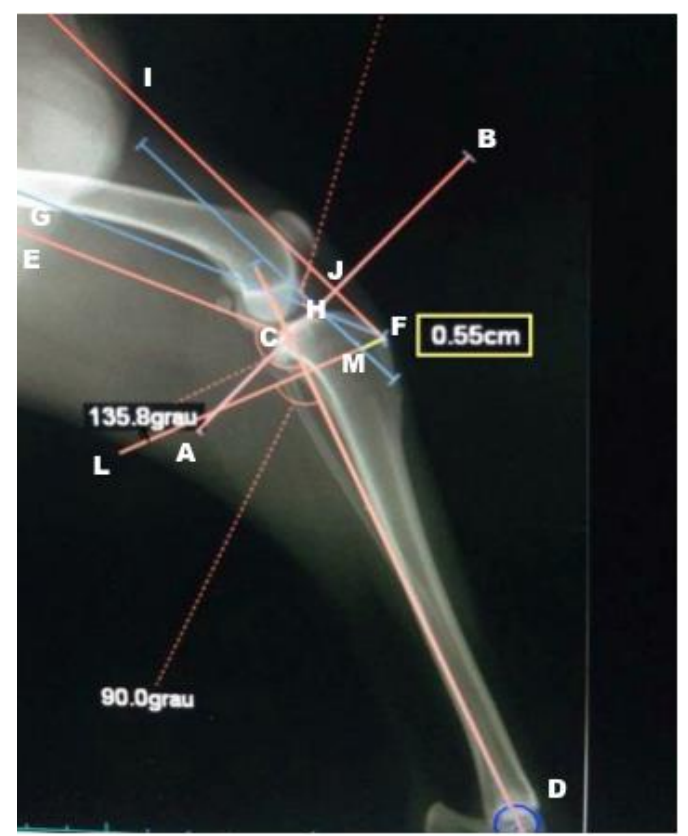

Figura 4. Método Ness (2011) de cálculo de avanço para TTA.

\section{Método de Koch (2016)}

Utilizando a régua do programa Workstation Fujifilm, o tamanho referente ao comprimento crânio caudal do platô tibial medial foi aferido, em seguida multiplicado pelo coeficiente 2,07, e uma nova reta com este comprimento foi traçada partindo da extremidade caudal do platô tibial através da inserção tibial do ligamento patelar. A parte da reta que ultrapassou a tuberosidade da tíbia corresponde a medida do avanço necessário (Figura 5).

Após todos estes cálculos acima terem sido realizados em ambos joelhos de cada animal, os resultados foram comparados e estatisticamente estudados quanto a ocorrência ou não de diferenças significantes entre os valores.

Os dados foram analisados descritivamente através de frequências absoluta e percentual para as variáveis categóricas e das medidas descritivas: 
média, desvio padrão, mediana e valores mínimo e máximo e foram analisados inferencialmente através de testes t-Student pareado $\mathrm{F}$ (ANOVA) para medidas repetidas e no caso de diferenças significantes foram utilizado testes de comparações múltiplas de Bonferroni e coeficiente de correlação de concordância com o respectivo intervalo de confiança. A margem de erro utilizada na decisão dos testes estatísticos foi de $5 \%$ e os intervalos foram obtidos com confiabilidade de $95 \%$.

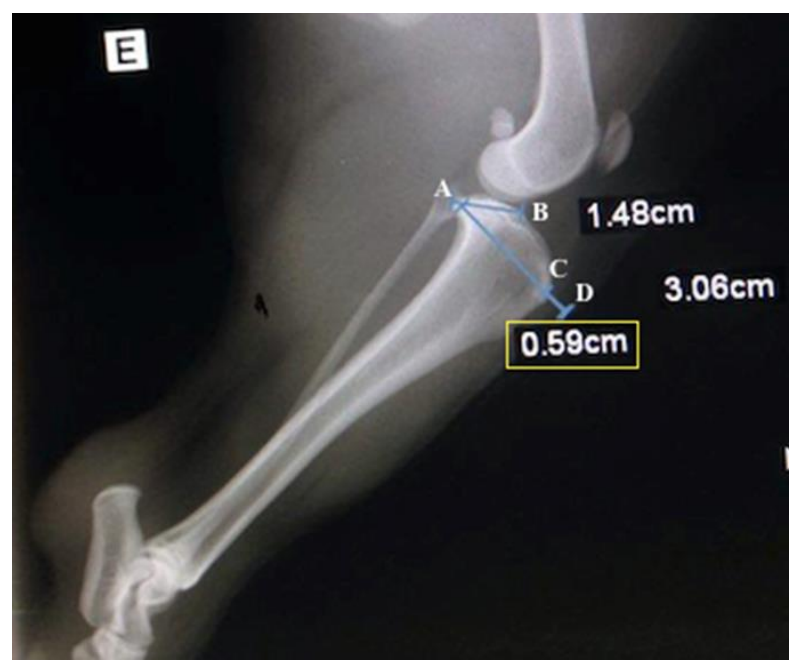

Figura 5. Método de cálculo de avanço para TTA segundo KOCH (2016). Onde $1.48 \mathrm{~cm}$ foi a medida do platô tibial (A-B) e $3.06 \mathrm{~cm} \mathrm{o}$ resultado de sua multiplicação pelo coeficiente 2.07, gerando a reta (A-D) e $0.59 \mathrm{~cm}$ o resultado do avanço necessário (C-D).

\section{Quadro Peso x Avanço (Vezzoni, 2010)}

Este método é uma correlação préestabelecida que relaciona o peso do animal e a quantidade de avanço necessária da tuberosidade da tíbia (Tabela 1). Esta tabela é baseada em um ângulo de platô tibial de 25 graus, podendo aumentar os valores descritos de avanço caso este ângulo aumente. Foi então correlacionado o peso de cada animal com a medida de avanço préestabelecida.

Após todos estes cálculos acima terem sido realizados em ambos joelhos de cada animal submetido ao estudo, os resultados foram comparados e estatisticamente estudados quanto a ocorrência ou não de diferenças significantes nos valores. Os valores obtidos também foram comparados com os tamanhos de implantes disponíveis no Brasil até o momento para as técnicas de TTA padrão e TTA rapid.
Tabela 1. Método do cálculo de avanço da tuberosidade da tíbia por medidas pré estabelecidas relacionadas ao peso dos animais.

\begin{tabular}{cc}
\hline $\begin{array}{c}\text { Tamanho do } \\
\text { espaçador }\end{array}$ & Peso do animal \\
\hline $3 \mathrm{~mm}$ & $<10 \mathrm{~kg}$ \\
$6 \mathrm{~mm}$ & $10-30 \mathrm{~kg}$ \\
$9 \mathrm{~mm}$ & $30-45 \mathrm{~kg}$ \\
$12 \mathrm{~mm}$ & $>45 \mathrm{~kg}$ \\
\hline
\end{tabular}

\section{Resultados e Discussão}

A escolha correta do tamanho do cage, obtida por um dos vários métodos de cálculo durante o período pré operatório é de extrema importância para o sucesso da técnica cirúrgica da TTA. Apesar de existirem diversos métodos de cálculo disponíveis na literatura, há poucos estudos (Millet et al., 2013; Cadmus et al., 2014; Meeson et al., 2018) que comparem precisão e eficiência, e incluam os métodos de Koch, Ness e do quadro peso-cage.

Nos joelhos direitos foi observado que as médias das medidas foram menos elevadas nos métodos do quadro pré definido $(3,9)$ e Tangente comum $(4,1)$ em relação aos outros métodos aplicados que variaram de 4,7 a 4,9, concordando com o estudo de Millet et al. (2013) quando afirmam que os valores observados no método da tangente comum foram baixos em relação aos valores encontrados no método do platô tibial. Comparando-se os métodos no lado esquerdo, a menor média correspondeu ao método do quadro pré definido $(3,9)$, e foi mais elevada $(5,2)$ pelo método descrito por Ness, 2011, variando de 4,3 a 4,9 nos outros três métodos.

Entre os lados, a única diferença significante $(\mathrm{p}<0,05)$ para a margem de erro fixada (5\%) foi registrada no método descrito por Ness (2011) que teve valor mais elevado no lado esquerdo do que no direito $(5,2 \times 4,9)$ (Tabela 1$)$, resultado que pode ser explicado pelo método apresentar um maior grau de dificuldade de realização e uma maior quantidade de pontos anatômicos a serem encontrados, aumentando sua variabilidade. Por não terem sido encontradas diferenças significanates entre os lados nos demais métodos, pode-se afirmar que este estudo vai de acordo com Vezzoni (2010) ao dizer que quando um membro apresentar luxação cranial da tíbia numa RLCCr, pode se utilizar o membro contralateral sadio para os cálculos do avanço.

Diferenças significantes entre os métodos foram registradas em ambos os lados $(p<0,001)$ e 
através dos métodos de comparações múltiplas se comprova diferenças significantes dos métodos Platô tibial, Koch, Ness em relação aos outros dois métodos: Tangente comum e Quadro pré definido em cada um dos lados (Tabela 2). Ness (2011) afirma que seria quase impossível realizar uma radiografia com angulação exata de 135 graus, o que influenciaria no cálculo da tangente comum, defendendo a lógica que a osteotomia da tíbia deve ser proveniente de pontos de referência exclusivo desse osso, como ocorre em seu método proposto e utilizado neste trabalho. O método citado por Koch (2016) usa somente a tíbia para os cálculos, porém o método de Hoffman et al. 2006 que se utiliza da articulação como um todo, e que também se faz necessária esta angulação de 135 graus, se mostrou sem diferença significante quando comparada aos métodos de Koch (2016) e Ness (2011). Esta diferença significante da tangente comum com a maioria dos métodos corrobora também com as afirmações de Millet et al. (2013) onde mencionam que a sofisticação relativa desse método pode predispor ao erro, visto que, em seu estudo, os avaliadores observaram diferenças no diâmetro do círculo da tíbia por não apresentar marcos pertinentes que padronizassem o desenho, podendo ser alterado consideravelmente, e que essa imprecisão altera o método da tangente comum.

Tabela 2. Estatísticas das medidas no grupo total.

\begin{tabular}{llll}
\hline Método & $\begin{array}{l}\text { Lado Direito } \\
\text { Média } \pm \text { DP }\end{array}$ & $\begin{array}{l}\text { Lado Esquerdo } \\
\text { Média } \pm \mathbf{D P}\end{array}$ & Valor de p \\
\hline Platô tibial & $0,47 \pm 0,18^{(\mathrm{A})}$ & $0,47 \pm 0,17^{(\mathrm{A})}$ & $\mathrm{p}^{(1)}=0,740$ \\
Tangente comum & $0,41 \pm 0,18^{(\mathrm{B})}$ & $0,43 \pm 0,18^{(\mathrm{B})}$ & $\mathrm{p}^{(1)}=0,382$ \\
Koch (2016) & $0,48 \pm 0,0^{(\mathrm{A})}$ & $0,49 \pm 0,19^{(\mathrm{A})}$ & $\mathrm{p}^{(1)}=0,555$ \\
Ness (2011) & $0,49 \pm 0,17^{(\mathrm{A})}$ & $0,52 \pm 0,18^{(\mathrm{A})}$ & $\mathrm{p}^{(1)}=0,038^{*}$ \\
Tabela & $0,39 \pm 0,15^{(\mathrm{B})}$ & $0,39 \pm 0,15^{(\mathrm{B})}$ & $\mathrm{p}^{(1)}=1,000$ \\
Valor $\mathrm{p}$ & $\mathrm{p}^{(2)<0,001^{*}}$ & $\mathrm{p}^{(2)}<0,001^{*}$ & \\
\hline
\end{tabular}

(*): Diferença significante a $5 \%$.

(1): Através do teste Student pareado.

(2): Através do teste F (ANOVA) para medidas repetidas com comparações de Bonferroni.

Obs. Se as letras entre parentêses são distintas se comprova diferença significante entre os métodos correspondentes.

Cadmus (2014) avaliaram métodos de cálculo para TTA comparando-se os métodos do platô tibial, da tangente comum, um template e um software simulador de TTA, observando-se uma variabilidade de tamanho do implante em $86 \%$, com diferença significante entre esses 4 métodos, indo de acordo com o presente estudo, onde também foram observadas diferenças significantes dos métodos Platô tibial e Tangente comum.

Quanto ao método do Quadro pré-definido, se pode atribuir o resultado deste estudo a sua subjetividade, por não levar em consideração variações anatômicas individuais da conformação da tuberosidade da tíbia, bem como animais com sobrepeso ou com peso abaixo da média que não foram excluídos do presente estudo. Em trabalho realizado por Meeson et al (2018) relatam que a conformação da tíbia influencia o grau de avanço e deve ser levada em consideração durante o planejamento.

Na tabela 3 se apresenta os coeficientes de concordância entre os pares de métodos por lado. Sendo classificado da seguinte forma: concordância pobre (ICC < 0,50), concordância moderada (ICC 0,51 - 0,70), concordância boa (ICC 0,71- 0,90) e concordância ótima (ICC > $0,91)$. Esta tabela mostra que os coeficientes de concordância foram de 0,60 (entre os métodos Koch 2016 e Quadro de valores pré-definidos no lado esquerdo) com uma concordância boa a 0,89 (Platô tibial e Ness (2011) do lado direito), valores estes que indicam uma concordância apropriada.

Em estudos realizados por Millet et al. (2013), foram observados baixos índices de concordância entre os métodos do platô tibial com o método da tangente comum. Tais resultados não foram observados nesta pesquisa, onde a 
comparação entre as duas técnicas mostrou um índice de concordância bom, apesar do limite inferior, porém ao comparar os demais métodos com o da tangente comum, pode-se observar, exceto no membro esquerdo, um índice de concordância moderado, indicando que este método é em conjunto com o método do quadro os que apresentam menor índice de concordância, inclusive quando comparados entre si.

Tabela 3. Coeficiente de correlação concordância entre os pares de métodos no total de animais.

\begin{tabular}{lll}
\hline Método & $\begin{array}{l}\text { Direito } \\
\text { Coeficiente }(\mathbf{I C} \text { 95\%) }\end{array}$ & $\begin{array}{l}\text { Esquerdo } \\
(\mathbf{I C} \text { 95\%) }\end{array}$ \\
\hline Platô tibial x Tangente comum & $0,71(0,53$ a 0,83$)$ & $0,83(0,71$ a 0,94$)$ \\
Platô tibial x Koch (2016) & $0,70(0,50$ a 0,83$)$ & $0,85(0,73$ a 0,92$)$ \\
Platô tibial x Ness (2011) & $0,89(0,80$ a 0,94$)$ & $0,81(0,68$ a 0,89$)$ \\
Platô tibial x Tabela & $0,65(0,46$ a 0,79$)$ & $0,64(0,45$ a 0,78$)$ \\
Tangente comum x Koch (2016) & $0,64(0,43$ a 0,79$)$ & $0,82(0,69$ a 0,90$)$ \\
Tangente comum x Ness (2011) & $0,65(0,45$ a 0,79$)$ & $0,67(0,49$ a 0,80$)$ \\
Tangente comum x Tabela & $0,69(0,50$ a 0,82$)$ & $0,65(0,43$ a 0,79$)$ \\
Koch (2016) x Ness (2011) & $0,80(0,67$ a 0,89$)$ & $0,78(0,62$ a 0,88$)$ \\
Koch (2016) x Tabela & $0,62(0,43$ a 0,76$)$ & $0,60(0,38$ a 0,74$)$ \\
Ness (2011) x Tabela & $0,68(0,51$ a 0,80$)$ & $0,65(0,47$ a 0,77$)$ \\
\hline
\end{tabular}

\section{Conclusão}

No presente estudo observou-se não existir diferença significantes entre os métodos do platô tibial, Ness e Koch, porém existindo entre eles em relação aos métodos da tangente comum e o do quadro com medidas pré estabelecidas. Também verificamos não haver diferença significante entre os lados direito e esquerdo em todos os métodos, exceto no método de Ness.

\section{Conflito de Interesse}

Os autores declaram não ter conflitos de interesse.

\section{Comitê de Ética}

O experimento foi aprovado pela Comissão de Ética no Uso de Animais (CEUA) da UFRPE, sob número de licença 102/2016.

\section{Referências}

Apelt, A.; Kowaleski, M.P.; Boudrieau, R.J. Effect of tibial tuberosity advancement on cranial tibial subluxation in canine cranial cruciate-deficient stifle joints: an in vitro experimental study. Vetetinary Surgery, 36(2): 170-77, 2007.

Cadmus, J.; Palmer, R.H.; Duncan, C. The effect of preoperative planning method on recommended tibial tuberosity advancement cage size. Veterinary Surgery, 43(8): 9951000, 2014.

Dennler, R.; Kipfer, N.M.; Tepic, S.; Hassig, M.; Montavon, P.M. Inclination of the patellar ligament in relation to flexion angle in stifle joints of dogs without degenera- tive joint disease. American Journal of Veterinary Research, 67(11): 1849-54, 2006.

Hoffmann, D.E.; Miller, J.M.; Lanz, O.I.; Martin, R.A.; Shires, P.K. Tibial tuberosity advancement in 65 canine stifles. Veterinary and Comparative Orthopedics and Traumatology. 19(4): 219-27, 2006.

Koch, D. An alternative measurement of spacer width in TTA surgery. Diessenhofen (Switzerland), 2016. Disponível em: https://dkoch.ch/fileadmin/user_upload/Publi kationsliste/Alternative_measurement_ofspac er_width_in_TTA_surgery_2016.pdf > Acesso em: 02 set. 2019.

Kowaleski, M.P.; Boudriew, R.J.; Pozzi, A. Stifle joint. In: Johnston, S.A.; Tobias, K.M. Veterinary surgery: small animal. Florida: Elsevier, 2017. p. 2926-3176.

Kuhn, K.; Ohlerth, S.; Makara, M.; Hassig, M.; Guerrero, T.G. Radiographic and ultrasonographic evaluation of the patellar 
ligament following tibial tuberosity advancement. Veterinary Radiology and Ultrasound, 52(4): 466-71, 2011.

Meeson, R.L.; Corah, L.; Conroy, M.C; Calvo, I. Relationship between tibial conformation cage size and advancement achieved in TTA procedure. Veterinary Research, 14(104): $1-7,2018$.

Miller, J.M.; Shires, P.K.; Lanz, O.L. Effect of 9 $\mathrm{mm}$ tibial tuberosity advancement on cranial tibial translation in the canine cruciate ligament-deficient stifle. Veterinary Surgery, 36(4): 335-40, 2007.

Millet, M.; Bismuto, C; Labruine, A.; Marin; B.; Filleur, A.; Pillard P., Sonet, J.; Cachon, T.; Etchepareborde, S. Reliability of the common tangent and tibial plateau methods measurement. Veterinary and Comparative
Orthopaedics and Traumatology, 26(6): 469-78, 2013.

Ness, M.G. OrthoFoam MMP Wedge for canine cruciate disease, West Yorkshire (UK): 2011. Disponível em: https://www.fourlimb.com.au/assets/brochure s/MMP054006.pdf>. Acesso em: 31 jul. 2019.

Vezzoni, L. TTA Preoperative Planning. Bologna (Italy): 2010, 61p. Disponível em: https://www.vezzoni.it/images/staff/CV/CV_ Vezzoni_Luca_long_Dec_2017.pdf >. Acesso em: 02 Set. 2019.

Zani, C.C.; Medeiros, R M.; Padilha Filho, J.G.; Machado, M.R.F.; Moraes, P.C., Feliciano, M.A.R. Hydromat action on bone healing in dogs submitted to technical tibial tuberosity advancement modified. Ars Veterinaria, 27(4): 205-10, 2011. 